\title{
Resistencia a antimicrobianos y evaluación del tratamiento combinado en la septicemia neonatal
}

\author{
María Espino Hernández, ${ }^{1}$ María Julia Couto Ramos, ${ }^{2}$ \\ Niurka Fiol Ferrer ${ }^{3}$ y Nidia Rojas Hernández ${ }^{4}$
}

RESUMEN Objetivos. Estudiar la resistencia a los antibióticos de las cepas bacterianas aisladas de pacientes con septicemia neonatal en un hospital ginecobstétrico de La Habana, Cuba, en el período comprendido entre enero de 1994 y diciembre de 1998, y evaluar el valor predictivo del método del "tablero de damas" (checkerboard) para seleccionar el tratamiento con combinaciones de antibióticos en pacientes graves.

Métodos. Se estudió a 229 neonatos, a quienes se les extrajo sangre para hemocultivo y estudios de sensibilidad a antimicrobianos. El estudio de la susceptibilidad de las cepas se realizó por el método de microdilución en caldo y se utilizó el tablero de damas en bandejas de caldo para evaluar la eficacia del tratamiento antimicrobiano combinado.

Resultados. Cincuenta por ciento de las cepas aisladas de Staphylococcus spp. coagulasanegativas y $37 \%$ de las cepas de S. aureus, principales agentes causales de sepsis neonatal, fueron resistentes a la meticilina. De ellas, 94,5\% resultaron resistentes a varios antibióticos. Cuatro cepas de Staphylococcus spp. coagulasa-negativas y una de Enterococcus faecium fueron resistentes a la vancomicina (concentración mínima inhibidora $=128 \mu \mathrm{g} / \mathrm{mL}$ ). Se observó una alta probabilidad de eficacia clínica $(P<0,01)$ con combinaciones de antibióticos que mostraron un efecto sinérgico in vitro, mientras que las reacciones antagónicas se correspondieron con la probabilidad altamente significativa de fracaso terapéutico clínico.

Discusión. La principal causa de septicemia neonatal en la Unidad de Cuidados Intensivos del Hospital "América Arias" en La Habana, Cuba, está constituida por las cepas de estafilococo resistentes a la meticilina y a otros antibióticos. En pacientes graves (atendiendo a lo establecido en el programa de política de este centro), la primera línea de tratamiento la constituye la combinación de un antibiótico aminoglucósido con un betalactámico. Este estudio demostró que la eficacia clínica de un tratamiento antibiótico combinado no está condiciona por el patrón de resistencia de la cepa y que se puede alcanzar, con una probabilidad elevada, un buen resultado clínico cuando se demuestra un efecto sinérgico in vitro.

Palabras clave Septicemia neonatal, resistencia microbiana, terapia combinada, tablero de damas.

La septicemia neonatal, ya sea temprana o tardía, es una de las principales causas de muerte de recién nacidos en todo el mundo y, por consiguiente, el factor que más

\footnotetext{
Escuela Latinoamericana de Medicina, Departamento de Agentes Biológicos, Ciudad de La Habana, Cuba. La correspondencia debe ser enviada a María Espino Hernández, a la siguiente dirección: Escuela Latinoamericana de Medicina, Departamento de Agentes Biológicos. Carretera Panamericana km 3,5, Santa Fe, Playa, Ciudad de La Habana. Cuba. Correo electrónico: mercedes@ elacm.sld.cu
}

2 Hospital Gineco-Obstétrico "América Arias", Departamento de Neonatología, Ciudad de La Habana, Cuba.

3 Hospital Gineco-Obstétrico "América Arias", Departamento de Microbiología, Ciudad de La Habana, Cuba.

4 Universidad de la Habana, Facultad de Ciencias Biológicas, Departamento de Microbiología, Ciudad de La Habana, Cuba. contribuye a elevar los índices de mortalidad infantil $(1,2)$.

Las maniobras terapéuticas a las que son sometidos algunos recién nacidos, ya sea en el momento del nacimiento o cuando requieren cuidados especiales, suelen ser en su mayoría invasoras. Esto, unido a los mecanismos de defensa prácticamente inexistentes a esta edad, ocasiona con frecuencia cuadros infecciosos muy graves. Estas 
infecciones son causadas generalmente por microbios de origen hospitalario, altamente resistentes a la mayoría de los antibióticos $(3,4)$. Lamentablemente, los esquemas de tratamiento aplicables al recién nacido séptico se reducen prácticamente al uso de antibióticos betalactámicos, aminoglucósidos y glucopeptídicos, lo que, aparejado al frecuente desarrollo de resistencia a los medicamentos antimicrobianos, dificulta mucho el tratamiento de estos pacientes.

En el Hospital "América Arias", de Ciudad de La Habana, es baja la mortalidad infantil. No obstante, la mayoría de los decesos se producen debido a infecciones bacterianas resistentes al tratamiento (datos de archivo y mapas microbiológicos del hospital no publicados). Esta es la causa principal que conduce a la frecuente aplicación indiscriminada $\mathrm{y}$ en ocasiones desacertada- de tratamientos con antibióticos de amplio espectro de acción, con todos los riesgos que ello implica.

La escasez de recursos que afronta el país y el alto costo de los antibióticos de última generación constituyen razones adicionales para establecer el control del uso de estos medicamentos a partir de la creación de estrategias locales.

En 1992, un grupo multidisciplinario de especialistas del hospital comenzó a elaborar una política de tratamiento basada en los recursos disponibles, lo que llevó al diseño de una nueva estrategia de diagnóstico clínicomicrobiológico. Como parte de esta estrategia se incorporó a la práctica clínica el método del "tablero de damas" (checkerboard) para el monitoreo del tratamiento combinado de antibióticos en pacientes graves o con evolución tórpida. Este estudio y uno anterior realizado por los propios autores (5) constituyen los primeros informes del empleo de esta técnica en Cuba.

El "tablero de damas", basado en la técnica de microdilución en caldo, fue diseñado hace algunos años y se incluye dentro de los métodos recomendados por el National Committee for Clinical Laboratory
Standards (NCCLS) de los Estados Unidos $(6,7)$.

Se han notificado estudios que evalúan mediante diferentes técnicas la sinergia in vitro de medicamentos contra diferentes especies bacterianas resistentes (8-10). Sin embargo, resultan escasas las experiencias clínicas que avalen paralelamente estos resultados.

En este estudio nos propusimos como objetivos: 1) analizar el cuadro epidemiológico de la unidad de cuidados intensivos neonatales (UCIN) del Hospital "América Arias" de Ciudad de La Habana, según los microorganismos vinculados a la sepsis y su resistencia a los medicamentos antimicrobianos de uso habitual en el servicio; 2) validar el empleo en nuestro medio del método del "tablero de damas" como elemento de predicción de la eficacia del tratamiento combinado con antibióticos; 3) identificar mediante este ensayo posibles alternativas terapéuticas, basadas en los recursos habitualmente existentes en el servicio, que puedan ser eficaces contra bacterias resistentes.

\section{MATERIALES Y MÉTODOS}

Se realizó un estudio de los casos consecutivos de sepsis neonatal que se presentaron en el Hospital Docente Gineco-Obstétrico "América Arias" durante el período comprendido entre enero de 1994 y diciembre de 1998. Este estudio abarcó a todos los recién nacidos internados en la UCIN con un diagnóstico de infección sistémica corroborado por estudios microbiológicos.

La población total estudiada fue de 229 pacientes. Con vistas a validar el empleo de la técnica del "tablero de damas" para predecir la eficacia del tratamiento combinado, se tomó una muestra formada por aquellos pacientes con un presunto diagnóstico de sepsis que fueron ubicados en camas previamente seleccionadas dentro del servicio (68 pacientes).

El tratamiento con antibióticos aplicado a todos los recién nacidos sépticos se aplicó según la política para el uso de antimicrobianos, establecida previamente en la UCIN.

\section{Política para el uso de antimicrobianos}

En este programa se definieron, entre otros aspectos, tres líneas principales de tratamiento escalonado. Estas líneas fundamentales se conformaron mediante la combinación de nueve antibióticos de uso habitual (cinco betalactámicos, tres aminoglucósidos y uno glicopeptídico) de la siguiente forma:

- Primera línea. Para emplear como terapia empírica en las sepsis tempranas: penicilina G-kanamicina, penicilina G-gentamicina. Para emplear como terapia inicial en las infecciones de inicio tardío: cefazolina o cefazolina-gentamicina.

- Segunda línea: penicilina G-amikacina, cefazolina-amikacina, azlocilinaamikacina, azlocilina-gentamicina.

- Tercera línea: ceftriaxona-gentamicina, ceftriaxona-amikacina, cefotaximagentamicina, cefotaxima-amikacina.

La vancomicina sola o con amikacina se reservó para las infecciones por estafilococos y enterococos multirresistentes que no respondieran a alguno de los tratamientos de segunda línea.

Otros tratamientos serían utilizados solo tras discutir el caso, dependiendo de los medicamentos existentes en ese momento.

\section{Procedimiento utilizado}

a) Extracción de sangre para cultivo (dos muestras siempre que fue posible) antes de la aplicación del tratamiento antimicrobiano.

b) Aplicación inmediata del tratamiento previsto según la política establecida en la UCIN. Este tratamiento se mantuvo durante 72 horas, siempre que el paciente se mantuviera clínicamente estable.

c) Aislamiento e identificación del agente causal de la infección, determinación del patrón de resistencia $\mathrm{y}$ definición in vitro del com- 
portamiento del microorganismo aislado ante el tratamiento combinado aplicado.

d) Evaluación a las 72 horas del tratamiento antimicrobiano aplicado, teniendo en cuenta los resultados microbiológicos obtenidos y el estado clínico del paciente.

En la indicación del hemocultivo, según lo descrito más arriba, se recogieron los siguientes datos: nombre del paciente, número de la historia clínica, fecha y hora de extracción de la muestra, y tratamiento antimicrobiano aplicado.

Los cambios de tratamiento que implicaran una línea terapéutica superior a las definidas dentro de la política establecida fueron discutidos previamente por el equipo de trabajo.

\section{Muestras}

Las muestras de sangre para cultivo se tomaron de las venas periféricas tras la desinfección de la zona. Se inocularon frascos de cultivo con $15 \mathrm{~mL}$ de caldo corazón estéril con $1,5 \mathrm{~mL}$ de sangre de cada paciente y se incubaron a $36 \pm 1^{\circ} \mathrm{C}$ en condiciones aeróbicas durante no menos de 12 horas ni más de 18 horas antes de realizar el primer subcultivo. El aislamiento y la identificación del agente causal se realizaron mediante métodos convencionales de laboratorio (11).

La obtención de un cultivo puro en el primer subcultivo se consideró como una muestra positiva al microorganismo cultivado. La presencia de bacterias grampositivas y gramnegativas habitualmente saprofitas, aisladas en subcultivos posteriores a una primera resiembra en la que no se obtuvo crecimiento microbiano, se consideró como indicio de una posible contaminación y el proceso se repitió. Al concluir el tratamiento, se tomó una nueva muestra de sangre para cultivo.

\section{Pruebas de susceptibilidad}

Paralelamente al proceso de identificación de los microorganismos se realizaron pruebas de susceptibilidad. El patrón de resistencia de las cepas se determinó mediante el método de microdilución para los antibióticos de uso en la UCIN: penicilina $G$, kanamicina, gentamicina, amikacina, cefazolina, cefotaxima, ceftriaxona, vancomicina y oxacilina (usado como marcador de la resistencia a la meticilina). Los valores de la concentración mínima inhibidora (CMI) se determinaron mediante procedimientos previamente establecidos (7). Se utilizaron reactivos de laboratorio estándares, de potencia conocida, para preparar las soluciones stock de los antibióticos, que fueron evaluados en concentraciones entre 0,125 y $128 \mu \mathrm{g} / \mathrm{mL}$. El control de la calidad se realizó mediante las cepas de referencia Staphylococcus aureus ATCC 29213, Escherichia coli ATCC 25922 y Pseudomonas aeruginosa ATCC 27853, según lo recomendado por el NCCLS.

\section{Pruebas de sinergia}

Las pruebas de sinergia se realizaron por el método del "tablero de damas". Para la configuración del panel de diluciones se partió de una concentración de ambos antibióticos de $128 \mu \mathrm{g} / \mathrm{mL}$ en las diferentes combinaciones probadas, y se evaluó la posibilidad de sinergia en combinaciones de los dos fármacos a diferentes concentraciones. Los resultados se evaluaron mediante el cálculo de los índices de la concentración fraccionaria inhibidora (índices CFI) (7):

- $\mathrm{CFI}_{\mathrm{A}}=\mathrm{CMI}_{\mathrm{A}}$ en la combinación/ $\mathrm{CMI}_{\mathrm{A}}$ solo.

- $\mathrm{CFI}_{\mathrm{B}}=\mathrm{CMI}_{\mathrm{B}}$ en la combinación/ $\mathrm{CMI}_{\mathrm{B}}$ solo.

- Índice $\mathrm{CFI}=\mathrm{CFI}_{\mathrm{A}}+\mathrm{CFI}_{\mathrm{B}}$.

Se consideró que había sinergia cuando se obtuvo un índice CFI $\leq 0,5$, sinergia baja o parcial cuando los valores de la CFI fueron $>0,5 \mathrm{y}<1$, adición o indiferencia cuando fueron $\geq$ $1 \mathrm{y}<2$, y antagonismo cuando fueron $=2$.

\section{Criterios para determinar la eficacia del tratamiento}

Con los resultados obtenidos en el laboratorio (cultivo, CMI y sinergia) y de acuerdo con la evolución clínica del paciente, el tratamiento aplicado fue evaluado según los siguientes criterios de eficacia:

- Tratamiento eficaz. Cuando al concluir el tratamiento, además de desaparecer los signos clínicos de infección no se obtuvo crecimiento microbiano en la muestra evolutiva del hemocultivo.

- Tratamiento ineficaz. Cuando fue necesario realizar cambios en el tratamiento iniciado, sustituyendo al menos uno de los fármacos de la combinación, o cuando, a pesar de haberse culminado el tratamiento, se detectó crecimiento del mismo agente causal con patrón de resistencia similar, acompañado o no de la persistencia de los signos y síntomas clínicos de infección.

El análisis de los resultados se realizó mediante tablas de contingencia de doble entrada. Para analizar las muestras pequeñas se utilizó la prueba exacta de Fisher (12). Para los cálculos se utilizó el paquete estadístico Microstat.

\section{RESULTADOS}

Durante los 5 años del período analizado (1994-1998) nacieron en la institución estudiada 16085 niños, 2948 de los cuales ingresaron a la UCIN. Durante este período se confirmaron 244 episodios de sepsis neonatal (15,1/1 000 nacidos vivos) en 229 recién nacidos (14,2/1000 nacidos vivos). El $10 \%$ de estos casos murieron por esta causa $(1,4 / 1$ 000), debida principalmente a bacterias gramnegativas $(60,9 \%)$. El cuadro 1 muestra los microorganismos vinculados a la sepsis según su frecuencia y la mortalidad asociada. 
CUADRO 1. Casos de sepsis neonatal según el agente causal (entre paréntesis el número de fallecimientos asociados). Hospital Gineco-Obstétrico "América Arias", La Habana, Cuba, enero de 1994 a diciembre de 1998

\begin{tabular}{|c|c|c|c|}
\hline Bacterias & $\begin{array}{l}\text { Infección } \\
\text { temprana }\end{array}$ & $\begin{array}{l}\text { Infección } \\
\text { tardía }\end{array}$ & $\begin{array}{c}\text { Total de } \\
\text { cepas aisladas }\end{array}$ \\
\hline \multicolumn{4}{|l|}{ Grampositivas } \\
\hline Staphylococcus spp. coagulasa-negativas & 51 & $49(2)$ & $100(2)$ \\
\hline Staphylococcus aureus & $51(4)$ & $11(1)$ & $62(5)$ \\
\hline Streptococcus spp. $\beta$-hemolíticas & $5(1)$ & - & $5(1)$ \\
\hline Enterococcus spp. & 8 & 5 & 13 \\
\hline \multicolumn{4}{|l|}{ Gramnegativas } \\
\hline Grupo BNFa & $17(2)$ & $10(3)$ & 27 (5) \\
\hline Enterobacter spp. & $14(1)$ & $7(3)$ & $21(4)$ \\
\hline Escherichia coli & $5(1)$ & $2(1)$ & $7(2)$ \\
\hline Klebsiella spp. & $2(1)$ & $2(1)$ & $4(2)$ \\
\hline Proteus mirabilis & 1 & 1 & 2 \\
\hline Citrobacter freundii & - & $1(1)$ & $1(1)$ \\
\hline Haffnia spp. & 1 & - & 1 \\
\hline Levaduras & - & $1(1)$ & $1(1)$ \\
\hline Total & $155(10)$ & $89(13)$ & $244(23)$ \\
\hline
\end{tabular}

a Bacilos no fermentadores.

Los resultados más relevantes obtenidos en las pruebas de susceptibilidad fueron:

- $93,8 \%$ y $96,7 \%$ de resistencia a penicilina G en Staphylococcus spp. coagulasa-negativas y $S$. aureus, respectivamente.

- $50 \%$ de los aislamientos de Staphylococcus spp. coagulasa-negativas y $37 \%$ de las cepas de $S$. aureus fueron resistentes a la meticilina, con valores para la oxacilina entre 4 y $128 \mu \mathrm{g} / \mathrm{mL}$ (mediana de $64 \mu \mathrm{g} / \mathrm{mL}$ ). De estas cepas, $94,5 \%$ presentaron resistencia cruzada a los antibióticos aminoglucósidos kanamicina y gentamicina y 32\% fueron también resistentes a la amikacina. En este grupo, 16 aislamientos mostraron susceptibilidad in vitro a la cefazolina.

- 4 cepas de Staphylococcus spp. coagulasa-negativas y 1 de Enterococcus faecium fueron resistentes a la vancomicina, todas con valores de CMI $\geq 128 \mu \mathrm{g} / \mathrm{mL}$ (cuadro 2).

- Resistencia elevada en enterobacterias -fundamentalmente las de los géneros Enterobacter y Klebsiella y la especie E. coli- a antibióticos aminoglucósidos y cefalosporinas en general, particularmente en los aislamientos de origen hospitalario $(\mathrm{CMI} \geq 128 \mu \mathrm{g} / \mathrm{mL}$ para cefalosporinas de tercera generación en $90 \%$ de las cepas).

- La amikacina fue el antibiótico que mostró la mayor eficacia in vitro $(57,1 \%$ de cepas susceptibles) y la cefazolina, la menor $(79,3 \%$ de aislamientos resistentes).

En los 68 pacientes se evaluaron 72 tratamientos. En el cuadro 3 se resume la eficacia clínica obtenida.

De los 72 estudios de sinergia realizados in vitro, 36 mostraron sinergia $(50 \%)$, con índices CFI entre 0,01 y 0,50 y una media geométrica de 0,29 . Se obtuvo sinergia baja o parcial en $19(26,4 \%)$, con índices CFI entre 0,51 y 0,75 y una media geométrica de 0,62 . En 12 los resultados fueron indiferentes o aditivos $(16,7 \%)$, con índice CFI = 1 en todos los casos. Cinco ensayos mostraron antagonismo (índice CFI = 2). El cuadro 4 presenta los resultados obtenidos en estas pruebas según los agentes causales y las combinaciones ensayadas.

Según el patrón de susceptibilidad de las cepas a los antibióticos usados en las combinaciones ensayadas, se observó que solo 5 de los 72 aislamientos fueron susceptibles a los dos antibióticos y en los 5 casos las pruebas in vitro mostraron sinergia; 28 aislamientos fueron susceptibles a uno de los dos antibióticos de la combinación y en estos casos se obtuvo sinergia en 18 pruebas (64,3\%), sinergia baja en $8(28,6 \%)$ e indiferencia o efecto aditivo en $2(7,1 \%)$. Un total de 39 cepas fueron resistentes a los dos antibióticos de la combinación ensayada;

CUADRO 2. Concentración mínima inhibidora de los antibióticos probados frente a los aislamientos de Staphylococcus spp. coagulasanegativas y Enterococcus faecium resistente a la vancomicina. Hospital Gineco-Obstétrico "América Arias", La Habana, Cuba, enero de 1994 a diciembre de 1998

\begin{tabular}{lcccccccrrrr}
\hline \multicolumn{1}{c}{ Aislamiento } & Fecha & $\mathrm{P}$ & $\mathrm{OX}$ & $\mathrm{AZ}$ & $\mathrm{K}$ & $\mathrm{G}$ & $\mathrm{AK}$ & $\mathrm{CZ}$ & $\mathrm{CTX}$ & CRO & VA \\
\hline Staphylococcus spp. & & & & & & & & & & \\
$\quad$ coagulasa-negativas & & & & & & & & & \\
Cepa 1 & $12 / 06 / 98$ & $\geq 128$ & $\geq 128$ & 32 & $\geq 128$ & 32 & 1 & $\geq 128$ & 32 & 64 & $\geq 128$ \\
Cepa 2 & $25 / 06 / 98$ & $\geq 128$ & $\geq 128$ & $\geq 128$ & $\geq 128$ & 32 & 1 & $\geq 128$ & 64 & $\geq 128$ & $\geq 128$ \\
Cepa 3 & $26 / 08 / 98$ & $\geq 128$ & $\geq 128$ & SP & $\geq 128$ & 32 & $\geq 128$ & 32 & $\geq 128$ & $\geq 128$ & $\geq 128$ \\
Cepa 4 & $14 / 11 / 98$ & $\geq 128$ & $\geq 128$ & SP & $\geq 128$ & $\geq 128$ & 32 & $\geq 128$ & $\geq 128$ & $\geq 128$ & $\geq 128$ \\
E. faecium & $10 / 09 / 97$ & 32 & $\geq 128$ & SP & 32 & 16 & $\geq 128$ & $\geq 128$ & $\geq 128$ & $\geq 128$ & $\geq 128$ \\
\hline
\end{tabular}

Nota: SP: sin probar; P: penicilina G; OX: oxacilina; AZ: azlocilina; K: kanamicina; G: gentamicina; AK: amikacina; CZ: cefazolina; CTX: cefotaxima; CRO: ceftriaxona; VA: vancomicina. 
CUADRO 3. Eficacia clínica de los tratamientos aplicados a los 68 pacientes en estudio. Hospital Gineco-Obstétrico "América Arias", La Habana, Cuba, enero de 1994 a diciembre de 1998

\begin{tabular}{lcrrrrr}
\hline & \multirow{2}{*}{$\begin{array}{c}\text { Total } \\
\text { Tratamiento }\end{array}$} & \multicolumn{2}{c}{ Eficaz } & & \multicolumn{2}{c}{ Ineficaz } \\
\multicolumn{1}{c}{ evaluados } & No. & $\%$ & & No. & $\%$ \\
\hline Penicilina G/kanamicina & 37 & 25 & 67,6 & & 12 & 32,4 \\
Penicilina G/gentamicina & 13 & 8 & 61,5 & & 5 & 38,5 \\
Penicilina G/amikacina & 5 & 3 & 60,0 & & 2 & 40,0 \\
Azlocilina/amikacina & 11 & 10 & 91,0 & & 9,0 \\
Cefazolina/amikacina & 1 & 1 & 100 & & 0 & 0 \\
Ceftriaxona/gentamicina & 1 & 1 & 100 & & 0 & 0 \\
Ceftriaxona/amikacina & 4 & 3 & 75,0 & & 1 & 25,0 \\
\hline
\end{tabular}

CUADRO 4. Resultados de las pruebas de sinergia in vitro. Hospital Gineco-Obstétrico "América Arias", La Habana, Cuba, enero de 1994 a diciembre de 1998

\begin{tabular}{|c|c|c|c|c|c|c|c|}
\hline \multirow{2}{*}{$\begin{array}{l}\text { Combinación } \\
\text { de antibióticos }\end{array}$} & \multirow[b]{2}{*}{ Pruebas } & \multirow[b]{2}{*}{ Agente causal } & \multirow[b]{2}{*}{ Cepas } & \multicolumn{4}{|c|}{ Sinergia in vitro } \\
\hline & & & & $S$ & SB & 1 & A \\
\hline \multirow{4}{*}{$\begin{array}{l}\text { Penicilina G } \\
\text { Kanamicina }\end{array}$} & \multirow[t]{4}{*}{37} & Staphylococcus & 26 & 11 & 6 & 8 & 1 \\
\hline & & Streptococcus & 1 & 1 & 0 & 0 & 0 \\
\hline & & Enterobacteriaceae & 8 & 0 & 2 & 2 & 4 \\
\hline & & Grupo BNFa & 2 & 1 & 1 & 0 & 0 \\
\hline \multirow{4}{*}{$\begin{array}{l}\text { Penicilina G } \\
\text { Gentamicina }\end{array}$} & \multirow[t]{4}{*}{13} & Staphylococcus & 9 & 4 & 4 & 1 & 0 \\
\hline & & Streptococcus & 1 & 1 & 0 & 0 & 0 \\
\hline & & Enterobacteriaceae & 2 & 2 & 0 & 0 & 0 \\
\hline & & Grupo BNF & 1 & 1 & 0 & 0 & 0 \\
\hline Penicilina G & \multirow[t]{2}{*}{5} & Spaphylococcus & 3 & 1 & 2 & 0 & 0 \\
\hline Amikacina & & Grupo BNF & 2 & 1 & 0 & 1 & 0 \\
\hline \multirow{3}{*}{$\begin{array}{l}\text { Azlocilina } \\
\text { Amikacina }\end{array}$} & \multirow[t]{3}{*}{11} & Staphylococcus & 7 & 5 & 2 & 0 & 0 \\
\hline & & Enterococcus & 2 & 1 & 1 & 0 & 0 \\
\hline & & Grupo BNF & 2 & 1 & 1 & 0 & 0 \\
\hline $\begin{array}{l}\text { Ceftriaxona } \\
\text { Amikacina }\end{array}$ & 4 & Enterobacteriaceae & 4 & 4 & 0 & 0 & 0 \\
\hline $\begin{array}{l}\text { Ceftriaxona } \\
\text { Gentamicina }\end{array}$ & 1 & Enterobacteriaceae & 1 & 1 & 0 & 0 & 0 \\
\hline Cefazolina & \multirow[t]{3}{*}{1} & \multirow[t]{3}{*}{ Staphylococcus } & 1 & 1 & 0 & 0 & 0 \\
\hline Total & & & 70 & 36 & 10 & 12 & 5 \\
\hline Iotal & & & 72 & 36 & 19 & 12 & 5 \\
\hline
\end{tabular}

Nota: S: sinergia $(\mathrm{CFI} \leq 0,5)$; SB: sinergia baja $(0,5<\mathrm{CFI}<1,0)$; I: indiferencia 0 adición $(1 \leq \mathrm{CFI}<2)$; $\mathrm{A}$ : antagonismo $(\mathrm{CFI} \geq 2)$. a Bacilos no fermentadores.

de ellas, 13 mostraron sinergia $(33,3 \%)$, 11 sinergia baja $(28,2 \%), 10$ indiferencia $(25,7 \%)$ y 5 antagonismo (12,8\%).

$\mathrm{Al}$ relacionar los resultados obtenidos en el laboratorio con los resultados clínicos se encontró que la eficacia del tratamiento fue de $88,9 \%$ cuando las pruebas in vitro mostraron sinergia $(P<0,01)$; de $68,4 \%$ cuando las pruebas reflejaron sinergia baja; de $66,7 \%$ cuando los resultados fueron indiferentes, y de $0 \%$ cuando fueron antagónicos $(P<$ $0,001)$.
Teniendo en cuenta la susceptibilidad de las cepas, la eficacia clínica del tratamiento fue de $100 \%$ cuando el microorganismo era sensible a los dos antibióticos de la combinación aplicada, de $78,6 \%$ cuando era sensible solamente a uno de ellos, y de $66,7 \%$ cuando era resistente a los dos. No se encontró diferencia estadística significativa entre los grupos.

De los 11 casos tratados con la combinación de azlocilina y amikacina, 6 estaban infectados por estafiloco- cos resistentes a la meticilina: 5 cepas de Staphylococcus spp. coagulasa- negativas resistentes a la meticilina (SCNRM) y 1 de $S$. aureus resistente a la meticilina (SARM). Se observó sinergia in vitro en 5 de los 6 ensayos, con una marcada disminución de los valores de la CMI con los dos antibióticos de la combinación. Los seis pacientes respondieron favorablemente a este tratamiento (cuadro 5).

\section{DISCUSIÓN}

El Hospital Docente GinecoObstétrico "América Arias" es uno de los mayores de este tipo de la capital cubana. Posee 273 camas y tiene a su cargo la atención de tres municipios muy poblados. En él se producen anualmente entre 3200 y 3500 nacimientos. Aunque la frecuencia de sepsis encontrada en este estudio fue superior a la notificada en los informes consultados, guarda relación con el bajo índice de mortalidad $(13,14)$. Esto se debe en parte a las diferencias existentes en el cuadro microbiológico asociado, con predominio, en nuestro caso, de bacterias grampositivas pertenecientes al género Staphylococcus, con aislamiento muy frecuente y baja mortalidad asociada, como puede apreciarse en el cuadro 1.

A lo largo de las dos últimas décadas, las diferentes especies del género Staphylococcus han constituido la principal causa de infección en las unidades de terapia neonatal. Las infecciones por SARM y SCNRM se han convertido desde entonces en las principales causas del incremento de la morbilidad por infecciones intrahospitalarias $(3,15)$. Desde principios del año 1992, cuando se iniciaron en el laboratorio de microbiología de este hospital los estudios para la determinación de la resistencia a la meticilina, se detectó que ya circulaban en la UCIN cepas de SCNRM. Por su parte, el SARM fue aislado por primera vez en 1994 y su prevalencia se ha incrementado paulatinamente dentro del servicio (16). No obstante, son principalmente las bacterias gramnegativas las asociadas a los casos mortales.

Dieciséis aislamientos de estafilococos resistentes a la meticilina mostraron 
CUADRO 5. Resultados in vitro e in vivo de la combinación de azlocilina y amikacina frente a cepas de Staphylococcus spp. coagulasa-negativas y resistentes a la meticilina (SCNRM) y $S$. aureus resistente a la meticilina (SARM). Hospital Gineco-Obstétrico “América Arias”, La Habana, Cuba, enero de 1994 a diciembre de 1998

\begin{tabular}{|c|c|c|c|c|c|c|c|}
\hline Aislamiento & No. & $\begin{array}{c}\mathrm{CMI}_{\mathrm{A} 1} \\
(\mu \mathrm{g} / \mathrm{mL})\end{array}$ & $\begin{array}{c}\mathrm{CMI}_{\mathrm{A} 2} \\
(\mu \mathrm{g} / \mathrm{mL})\end{array}$ & $\mathrm{CMI}_{\mathrm{A} 1} / \mathrm{CMI}_{\mathrm{A} 2}$ & $\begin{array}{l}\text { Índice } \\
\text { CFI }\end{array}$ & Resultado & $\begin{array}{c}\text { Tratamiento } \\
\text { eficaz }\end{array}$ \\
\hline SCNRM $^{a}$ & 5 & & & & & & \\
\hline Cepa 1 & & $\geq 128$ & 32 & $32 / 16$ & 0,75 & SB & Sí \\
\hline Cepa 2 & & $\geq 128$ & $\geq 128$ & $2 / 0,06$ & 0,015 & $S$ & Sí \\
\hline Сepa $3^{a}$ & & 128 & 1 & $32 / 0,25$ & 0,5 & S & Sí \\
\hline Cepa 4 & & 16 & 32 & $2 / 1$ & 0,156 & $S$ & Sí \\
\hline Cepa 5 & & 8 & 64 & $2 / 4$ & 0,312 & $S$ & Sí \\
\hline SARM & 1 & 64 & 32 & $16 / 8$ & 0,5 & $S$ & Sí \\
\hline
\end{tabular}

Nota: A1: azlocilina; A2: amikacina; CMl: concentración mínima inhibidora; CFI: concentración fraccionaria inhibidora; SB: sinergia baja; S: sinergia

a SCNRM resistente a la vancomicina.

susceptibilidad in vitro a la cefazolina. Este hallazgo coincidió con valores de CMI límites para la oxacilina $(4-8 \mu \mathrm{g} / \mathrm{mL})$. Atendiendo a que la resistencia de los estafilococos a la meticilina implica resistencia a todos los antibióticos betalactámicos, podría tratarse en estos casos de cepas hiperproductoras de $\beta$ lactamasa (15). Sin embargo, dada la evolución de la expresión fenotípica que están sufriendo estos microorganismos (17, 18), no se puede descartar que se trate realmente de agentes portadores del gen mec $A$, aunque este aspecto no fue objetivo de este estudio. Krediet et al. (19) informaron el hallazgo de cepas de Staphylococcus spp. coagulasa-negativas portadoras del gen mec $A$ susceptibles in vitro a la cefazolina, con una respuesta satisfactoria a este tratamiento en los pacientes infectados.

$\mathrm{Si}$ bien las cepas de estafilococos sensibles a la meticilina también se mostraron sensibles a la mayoría de los antibióticos probados, las cepas de SARM y SCNRM pusieron en evidencia su carácter multirresistente. En este estudio se encontraron cuatro cepas de SCNRMy una de Enterococcus faecium resistentes a la vancomicina (cuadro 2). Los dos primeros aislamientos se produjeron en el mes de junio de 1998, con 14 días de diferencia; ambas infecciones fueron adquiridas en la UCIN y los pacientes fueron recién nacidos prematuros, uno de ellos con peso al nacer $<1500$ g, que presentaron síntomas muy marcados. Con anterioridad ya se había aislado en la UCIN una cepa de enterococo resistente a la vancomicina y quizás este haya sido el punto de partida de la resistencia encontrada posteriormente en estafilococos, probablemente por la transferencia horizontal del gen van $A$ (20). En el mismo año 1998 se produjeron los otros dos aislamientos, esta vez no coincidentes en el tiempo y, como en los casos anteriores, afectaron a recién nacidos prematuros de bajo peso al nacer.

El hallazgo de enterococos resistentes a la vancomicina se notificó por primera vez a mediados de la década de 1980 y hasta la fecha su aislamiento se ha incrementado con características epidémicas, principalmente en los Estados Unidos (21). La cepa aislada en este estudio tiene características similares a las de las cepas que circulan en los Estados Unidos por su patrón de resistencia bastante amplio (para los antibióticos probados), en lo que difiere de los aislamientos procedentes de Europa, cuya resistencia a la vancomicina no se acompaña de resistencia a otros compuestos (22). La mayoría de los aislamientos de este germen se produjeron en los dos últimos años del estudio, por lo que se hace necesario establecer en el servicio un sistema para la vigilancia de su circulación, pues puede convertirse rápidamente en una verdadera emergencia.

En general, las bacterias gramnegativas presentaron una gran resistencia a los antibióticos probados, aunque la mayor dificultad en la UCIN no se debe a su resistencia, sino a la liberación de sus potentes endotoxinas, que en no pocas ocasiones llevan al recién nacido a un estado de choque irreversible.

El tratamiento combinado con un antibiótico betalactámico y un aminoglucósido suele ser eficaz en el tratamiento de infecciones por diversos agentes causales, pero la posibilidad de que el betalactámico inactive al aminoglucósido es un hecho probado (23).

La política para el uso de antimicrobianos desarrollada en el hospital se justifica, dados la escasez de estos recursos y el creciente desarrollo de resistencia a los antibióticos que se observa en muchas especies bacterianas. Por lo tanto, este programa de tratamiento se conformó tomando en consideración los antibióticos habitualmente disponibles.

En el período 1993-1994, el laboratorio de este centro asistencial evaluó la eficacia de la combinación de penicilina G y kanamicina en 63 cepas de estafilococos coagulasa-positivas $y$ coagulasa-negativas procedentes de recién nacidos sépticos (5) y los resultados obtenidos permitieron que esta combinación se utilizara para el tratamiento empírico de la sepsis temprana hasta el año 1996. A partir de ese momento y por acuerdo del Comité Farmacoterapéutico del hospital, la kanamicina fue sustituida por la gentamicina debido a su elevada toxicidad y su débil acción, principalmente frente a bacterias gramnegativas.

De cualquier forma, como se puede apreciar en el cuadro 3, ambas combinaciones presentaron una eficacia clínica algo superior a $60 \%$ en los pacientes evaluados. Esta eficacia puede considerarse buena para una terapia empírica, si se tiene en cuenta la elevada resistencia encontrada en las cepas de mayor circulación en el servicio. 
En este estudio se observaron resultados antagónicos con la combinación penicilina G-kanamicina en 5 pruebas frente a diferentes especies microbianas, 4 de ellas pertenecientes a la familia Enterobacteriaceae (cuadro 4). Estas cepas tenían en común que eran resistentes a los dos antibióticos de la combinación ensayada (CMI $\geq 128 \mu \mathrm{g}$ / $\mathrm{mL}$ para ambos antibióticos en los 5 casos). Según informes consultados (24), altos niveles de resistencia a antibióticos aminoglucósidos (CMI = $2000 \mu \mathrm{g} / \mathrm{mL}$ ) limitan o suprimen la sinergia en la combinación con antibióticos betalactámicos y, aunque en este trabajo no se evaluó la sensibilidad de los aislamientos a concentraciones $>128$ $\mu \mathrm{g} / \mathrm{mL}$, en cepas resistentes se obtuvo menos sinergia. No obstante, con cepas resistentes a los dos antibióticos de la combinación y resistencia adicional a otros compuestos, se obtuvo sinergia completa o parcial en 13 y 11 ensayos, respectivamente.

Al analizar los resultados clínicos se encontró un 88,9\% de eficacia del tratamiento en casos con resultados sinérgicos in vitro, cifra aceptable si se consideran los múltiples factores que pueden afectar a la respuesta in vivo (25). En las pruebas con resultados indiferentes o aditivos se encontró un $66,7 \%$ de eficacia clínica. En la mayoría de estos ensayos se observó una disminución sustancial de la CMI del aminoglucósido de la combinación, mientras que la CMI del betalactámico permanecía inalterada. Cabría preguntarse si esta observación no pasa de ser una simple curiosidad de laboratorio, propia del método empleado, o si el betalactámico - a pesar de su resistencia in vitro- de algún modo debilita la pared celular bacteriana y permite la entrada del antibiótico aminoglucósido, lo que provocaría finalmente la muerte bacteriana por sinergia.

Los casos en los que se encontró un efecto antagónico in vitro se correspondieron con fracasos terapéuticos, lo que confirma que el método puede predecir con bastante exactitud las combinaciones de antibióticos inadecuadas para una determinada infección (23).

Algunas combinaciones de antibióticos han sido ensayadas como tratamientos alternativos a la terapia con vancomicina en infecciones por SARM, SCNRM y enterococos multirresistentes $(9,10,24)$. Antes de poder contar con la vanomicina, en la UCIN del hospital se habían aplicado empíricamente diferentes tratamientos. Los buenos resultados obtenidos con la combinación de azlocilina y amikacina aplicada a un paciente infectado por una cepa de $S$. haemolyticus multirresistente fue el punto de partida para su evaluación y uso posterior (26). Los resultados obtenidos con los 6 casos aquí presentados (cuadro 5) constituyen una nueva muestra del posible alcance de esta combinación de antibióticos, por lo que sería conveniente profundizar en los estudios con cepas que posean diferentes patrones $\mathrm{y}$ niveles de resistencia.

Según los resultados observados en las pruebas de sinergia, se puede afirmar que un resultado sinérgico in vitro de un betalactámico y un aminoglucósido podría predecir un resultado clínico satisfactorio con la combinación ensayada $(P<0,01)$, mientras que cuando los resultados son antagónicos, las probabilidades de fracaso terapéutico clínico son mayores $(P<0,001)$. Además, la respuesta clínica al tratamiento no está condicionada por la resistencia que muestre el microorganismo a los antibióticos de la combinación por separado, de lo que se infiere $-y$ fue demostrado en este estudio- que también pueden lograrse buenos resultados clínicos en pacientes infectados por cepas resistentes, si se demuestra un efecto sinérgico —o al menos no antagónico-en las pruebas in vitro.

A pesar de las dificultades que aún existen para la estandarización y reproducibilidad del método del "tablero de damas" (7), su empleo como respaldo al tratamiento, como lo demuestra esta investigación, proporciona una amplia cobertura de seguridad para tratamientos con antibióticos en pacientes graves infectados por cepas resistentes. Por otra parte, es necesario destacar que, además del dato cualitativo del resultado de la interacción entre los fármacos, el método proporciona información sobre las concentraciones óptimas de ambos antibióticos con las que ocurre la máxima interacción sinérgica, lo cual permite adecuar las dosis de los fármacos a las características particulares de cada paciente. Esto garantiza una menor probabilidad de efectos indeseables cuando los productos utilizados tienen elevada toxicidad.

Adicionalmente, con este estudio se evidencia la posibilidad de rescatar algunos medicamentos de generaciones anteriores para el tratamiento de infecciones graves en los recién nacidos.

Agradecimentos. Agradecemos a todo el personal médico del Servicio de Neonatología del Hospital GinecoObstétrico "América Arias" su colaboración, y en especial a las doctoras Sonia Ferrás, Neyda Cendán y Alina Ferreiro, su apoyo en la evaluación de los pacientes. A la Dra. Natasha Páez, Jefa del Servicio de Microbiología, se le agradece el apoyo brindado para el mejor desarrollo del trabajo.

\section{REFERENCIAS}

1. Schmidt BK, Kirpalani HM, Corey M, Low DE, Philip AGS, Ford-Jones EL. Coagulasenegative staphylococci as true pathogens in newborn infants: a cohort study. Pediatr Infect Dis J 1987;6:1026-1031.

2. Montero RA, Álvarez TM, Barbadillo FI, Carpintero IM, Sastre EH, Alonso BA. Análisis de sepsis por estafilococo coagulasa negativo en una unidad neonatal. Acta Pediatr Esp 1998;56:182-186.

3. Kollef MH. Antibiotic resistance among Grampositive bacteria in the hospital setting: what can we do about it? [Sitio en Internet] Medscape, Inc. Disponible en http://www. medscape.com/viewprogram/623. Acceso el 10 de diciembre de 2002.

4. Wolff MJ. Use and misuse of antibiotics in Latin America. Clin Infec Dis 1993;17(Supl 2): S346-S351.

5. Espino M, Couto MJ, Lee M, Páez N, Meriño E. Efecto sinérgico de penicilina $G$ y kanamicina 
en septicemia neonatal por estafilococos. Rev Cubana Pediatr 1995;67:155-164.

6. Berenbaum MC. A method for testing for synergy with any number of agents. J Infect Dis 1978;137:122-130.

7. National Committee for Clinical Laboratory Standards. Method for dilution antimicrobial susceptibility test for bacteria that grow aerobically. Wayne, Pa.: NCCLS; 1993. (Document M7-A3, Supplement 1).

8. Matsumoto Y. Combination cefixime/amoxicillin against penicillinresistant Streptococcus pneumoniae infection. Chemotherapy 1998;44 (Suppl 1):6-9.

9. Otsuka Y, Yoshibe T, Namioka M, Ezaki T. Combination effect of teicoplanin and betalactams on MRSA. Jpn J Antibiot 2000;53: 643-651.

10. Kiri N, Archer G, Climo MW. Combination of lysostaphin with beta-lactams is synergistic against oxacillin resistant Staphylococcus epidermidis. Antimicrob Agents Chemother 2002; 46:2017-2020.

11. Koneman EW, Allen SD, Dawell VR, Jauda WM, Sammers HM, Winn WC. Diagnóstico microbiológico. Texto y atlas a color. 3. ${ }^{\mathrm{a}}$ ed. México, DF: Panamericana; 1992.

12. Sigarroa A. Biometría y diseño experimental. La Habana: Pueblo y Educación; 1985.

13. Rodríguez JC, Fraga JM, García RC, Fernández-Lorenzo JR, Martínez IC. Sepsis neonatal: indicadores epidemiológicos en relación con el peso del recién nacido y el tiempo de hospitalización. An Esp Pediatr 1998;48:401-408.

14. Leibovitz E, Fidel-Rimon O, Juster-Reicher A, Amitay M, Miskin A, Barak Y, et al. Sepsis at a neonatal intensive care unit: a four year retrospective study (1989-1992). Isr J Med Sci 1997;33:734-738.

15. Brumfitt W, Hamilton-Miller JMT. The challenge of methicillin-resistant Staphylococcus aureus. Drugs Exp Clin Res 1994;20:215-224.

16. Niebla A, Espino M, González I, Otero I, Lemus M, González L, et al. Oxacillin resistance in Staphylococcus clinical isolates from Havana City Hospital, Cuba. Rev CENIC 2001;32:33-36.

17. Torvaldsen S, Roberts C, Riley TV. The continuing evolution of methicillin-resistant Staphylococcus aureus in Western-Australia. Infect Control Hosp Epidemiol 1999;20:133135.

18. Asada K, Inoba Y, Tateda E, Kuwahara AK, Ito I, Imaratsu K. Evolution and resistance expression of MRSA isogenic strains with different types of phenotypic expressions. Acta Biochem Pol 1995;42:517-524.

19. Krediet TG, Jones ME, Gerards LJ, Fleer A. Clinical outcome of cephalothin versus vancomycin therapy in the treatment of coagulase-negative staphylococcal septicemia in neonates: relation to methicillin resistance and mec $A$ gene carriage of blood isolates. Pediatrics 1999;103:E29.

20. Noble WC, Virani Z, Cree RGA. Co-transfer of vancomycin and other resistance genes from
Enterococcus faecalis NCTC 12201 to Staphylococcus aureus. FEMS Microbiol Lett 1992;72:195-198.

21. Martone WJ. Spread of vancomycin-resistant enterococci: why did it happen in the United States? Infect Control Hosp Epidemiol 1998; 19:539-545.

22. Goossens H. Spread of vancomycin-resistant enterococci: differences between the United States and Europe. Infect Control Hosp Epidemiol 1998;19:546-551.

23. Holm SE. Interaction between beta-lactam and other antibiotics. Rev Infect Dis 1986;8 (Supl 3):S305-S314

24. Predari SC, Gutiérrez MA, Ribas C, Molinari GS, Santoianni JE. Susceptibility of Enterococcus faecalis to twelve antibiotics, time-kill assays and high-level aminoglycoside resistance in a university hospital in Argentina. Rev Argent Microbiol 1991;23:67-78.

25. Moellering RC Jr. Antimicrobial synergism: an elusive concept. J Infect Dis 1979;140:639-641.

26. Espino M, Fiol N, Lee M, Couto MJ. Tratamiento con azlocilina y amikacina en sepsis neonatal por Staphylococcus haemolyticus multirresistente. Rev Cubana Pediatr 2000;72: 60-64.

Artículo recibido el 3 de abril de 2002. Aceptado para publicación, tras revisión, el 15 de noviembre de 2002.
ABSTRACT

\section{Resistance to antimicrobials and combination therapy assessment in neonatal sepsis}

Objectives. To estimate antibiotic resistance among bacterial strains isolated from patients with neonatal sepsis from January 1994 through December 1998, and to assess the predictive value of the checkerboard method for selecting treatment with combination antibiotic therapy in seriously ill patients.

Methods. The study of strain susceptibility was carried out by microdilution in broth, and the checkerboard method in broth trays was used to assess the efficacy of antibiotic combination therapy.

Results. Fifty per cent of the strains of coagulase-negative Staphylococcus spp. and 37\% of $S$. aureus strains, which are the main pathogens involved in neonatal sepsis, were resistant to methycillin. Of these strains, $94.5 \%$ were resistant to several antibiotics. Four strains of coagulase-negative Staphylococcus spp. and one strain of Enterococcus faecium were resistant to vancomycin (minimal inhibitory concentration $=128 \mu \mathrm{g} / \mathrm{mL}$ ). We noted a high probability of clinical efficacy $(P<0.01)$ with antibiotic combinations having a synergistic effect in vitro, whereas antagonic reactions were seen in close association with a highly significant probability of clinical therapeutic failure.

Discussion. The main cause of neonatal sepsis in the Neonatal Intensive Care Unit at "América Arias" Hospital in Havana, Cuba, were strains of Staphylococcus resistant to methycillin and other antibiotics. In seriously ill patients (according to policy established at this institution), the first line of treatment is a combination of an aminoglycoside antibiotic and a beta-lactamic antibiotic. This study showed that the clinical efficacy of combination antibiotic therapy is not determined by the resistance pattern of the strain and that good clinical results can be attained, in all likelihood, when a synergistic effect can be shown in vitro. 